\title{
Investigation of wear behavior of nanoalumina and marble dust-reinforced dental composites
}

https://doi.org/10.1515/secm-2017-0152

Received April 30, 2017; accepted May 24, 2018; previously published online August 14, 2018

\begin{abstract}
In the present work, the effects of adding nanoalumina and marble dust on the wear behavior of dental composites were investigated. The hardness of dental composite was determined using Vickers micro-hardness tester. A two-body abrasive wear test was performed on the dental wear simulator under the medium of artificial saliva. The experiments were performed as per the Taguchi orthogonal array and steady state condition by varying parameters such as filler content, normal load, sliding velocity, and number of cycles. The hardness results indicated that the incorporation of $5 \mathrm{wt}$. \% of nanoalumina increased the hardness of the dental composite by $12 \%$, whereas the incorporation of $5 \mathrm{wt}$. \% of marble dust increased the hardness of the dental composite by $7 \%$. Also, for the experiments as per the Taguchi orthogonal array, the mean volumetric wear in the case of nanoalumina-filled dental composite was $9.6 \%$ less than that of marble dust-filled dental composite. However, in both the cases, the volumetric wear increased with the increase in normal load, sliding speed, and number of cycles but decreased with the increase in filler content. Analysis of variance (ANOVA) of the results indicated that normal load was less significant compared to filler content, sliding speed, and number of cycles.
\end{abstract}

Keywords: dental composite; marble dust; nanoalumina; Taguchi method; wear.

\section{Introduction}

The study of wear behavior of dental materials is one of the important factors in understanding the deterioration level under clinical conditions. The excessive differential wear of teeth and restorative materials has significant deleterious effects on the biologic, functional, and aesthetic condition of the masticatory system. Hence, in this

\footnotetext{
*Corresponding author: Shiv Ranjan Kumar, Mechanical Engineering Department, JECRC University, Jaipur, India, e-mail: ranjan.shiv@gmail.com
}

Anoj Meena, Harlal Singh Mali and Amar Patnaik: Mechanical Engineering Department, M.N.I.T Jaipur, India

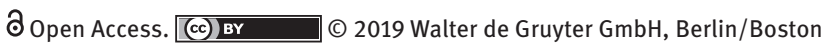

regard, the controlled investigations of various factors that might influence tooth and restoration wear have been performed to date. These examinations include the study of the effects of thermo-cycling [1], cyclic loading [2, 3], applied load [4], degree of polymerization [5], aging [6], water absorption [7], lubricant $\mathrm{pH}[8,9]$, surface coatings $[10,11]$, tooth brushing/dentifrices [12], and silanization [13-15] on the wear of dental material. The wear assessments for these studies were based on two-body and three-body wear simulation methods. However, the major loss in dental material is due to abrasion and erosion phenomena in the oral environment. It has been documented that the effect of filler volume on wear resistance follows a linear relationship, with high filler volumes decreasing wear rates [16]. In dental implantology, the alumina was initially used by the dental material scientist mainly in screws, anchorage elements, pin implants, and crowns, etc. [17, 18]. The application of alumina as a filler in dental materials provided appropriate aesthetic properties, chemical and biological inertness, high mechanical wear resistance, and overall long-term stability [19].

Currently, most commercial dental composite resins contain inorganic nanoparticles to achieve enhanced performance. These improvements are suggested to arise from the high specific surface area and rich surface functional groups of the nanoparticles [20]. Although these nanoparticles have substantially improved composite properties including abrasion resistance, polymerization shrinkage, and mechanical strength, more progress is still needed to achieve long-term satisfactory restorations for clinical therapy [21, 22]. In Rajasthan, India, one of the serious concerns is how to utilize marble dust or slurry. A very large amount of marble is being dumped by marble cutting industries in any nearby pit or vacant spaces. This imposes the biggest threats to nature and human health. Simultaneously, it produces challenges to the environment in terms of the topographic alteration, land occupation, surface and ground water contamination, and air pollution [23-25]. Therefore, utilization of marble dust in the production of new materials will help to protect the environment. Recently, the use of marble dust as replacement materials was investigated by various researchers [26-30]. Therefore, recycling and reuse of industrial wastes play a vital role in both solving the industrial waste problem and in getting benefit from it. Marble dust is used as one 
of filler ingredients that can adequately and reliably contribute to the performance of dental composite materials. Apart from the performance aspect, marble dust will greatly reduce the cost of artificial teeth materials.

The aim of the current study was to evaluate the wear performance of dental composite materials filled with nanoalumina and marble dust filler in an artificial saliva medium under steady state conditions and the Taguchi experimental design.

\section{Materials and methods}

\subsection{Materials}

Two different particulate filler materials such as nanoalumina and commercially available marble dust were used in this study. The properties of nanoalumina (particle size $20 \mathrm{~nm}$, high purity 98\%) and marble dust (particle size $70 \mathrm{~mm}$, Grade Makrana Marble, Udaipur, India) are presented in Tables 1 and 2, respectively. Monomer bisphenol-A glycidyl methacrylate (BisGMA) was procured from Esstech Inc. (Essington, PA, USA). The diluent tri-ethylene glycol dimethacrylate (TEGDMA) was purchased from TCI (Tokyo, Japan). Photo initiator camphorquinone (CQ) was purchased from Spectrochem (Mumbai, India). 2-dimethylaminoethyl methacrylate (DMAEMA), butylated hydroxytoluene (BHT) and coupling agent methacryloxy propyltrimethoxy silane (MPS) were purchased from Sigma Aldrich (Bangalore, India). For two-body wear assessment, artificial saliva was used, and the composition is presented in Table 3.

\subsection{Fabrication of dental composite}

The matrix material consists of resin matrix, e.g. BisGMA (52 wt. \%) and TEGDMA (45 wt. \%) and were mixed

Table 1: Properties of aluminum oxide nanoparticles.

\begin{tabular}{ll}
\hline Properties & Metric \\
\hline Density & $3.85 \mathrm{~g} / \mathrm{cm}^{3}$ \\
Molar mass & $101.96 \mathrm{~g} / \mathrm{mol}$ \\
Melting point & $2100^{\circ} \mathrm{C}$ \\
\hline
\end{tabular}

Table 2: Properties of marble dust.

\begin{tabular}{ll}
\hline Properties & Metric \\
\hline Fineness/fineness modulus & $1.5 \mathrm{~m}^{2} / \mathrm{g}$ \\
Specific gravity & 2.67 \\
\hline
\end{tabular}

Table 3: Medium used for wear analysis in the present study.

\begin{tabular}{|c|c|c|c|}
\hline Medium & Marketed by & $\begin{array}{l}\text { Brand } \\
\text { name }\end{array}$ & Composition \\
\hline $\begin{array}{l}\text { Artificial } \\
\text { saliva }\end{array}$ & $\begin{array}{l}\text { Global Dent Aids Pvt. } \\
\text { Ltd., New Delhi, India }\end{array}$ & Saleva & $\begin{array}{l}\text { Water, sorbitol, } \\
\text { glycerin, gum, } \\
\text { sodium saccharin, } \\
\text { sodium phosphate, } \\
\text { cetylpyridinium } \\
\text { chloride, brilliant blue }\end{array}$ \\
\hline
\end{tabular}

together with the polymerization initiator and accelerator CQ, DMAEMA, and BHT. Initially, the fillers nanoalumina and marble dust were mixed with silane (MPS) and water solutions and stirred uniformly up to $6 \mathrm{~h}$. Nine different composites were prepared by varying weight percentages of silane-treated nanoalumina and marble dust, respectively. Composites were prepared by pouring a blend mixture into a mold of the desired dimensions. The compositions of composite resins are presented in Table 4.

\subsection{Vickers micro-hardness test}

The nanoalumina- and marble dust-filled dental composite specimens of dimensions 8-mm diameter and 6-mm length were fabricated in the glass mold. After curing was done, the specimens were placed in distilled water for $24 \mathrm{~h}$ to ensure complete curing. Then, the specimens were subjected to 500-g load up to 30-s test on the machine microhardness tester (UHL VMHT, Walter, UK). Six indentations were made on the surface of the sample. The mean value (d) of the six indentations on each sample was considered. The Hv values were calculated in accordance to the ASTM E384-11e1 standard using Eq. (1).

$$
\mathrm{Hv}=1854.4 \times \frac{\mathrm{F}}{\mathrm{d}^{2}}
$$

Table 4: Detailed composition of unfilled and particulate-filled dental composites.

\begin{tabular}{llll}
\hline Material & Resin & $\begin{array}{l}\text { Filler } \\
\text { content }\end{array}$ & Filler type and size \\
\hline DC-0 & BisGMA (52 wt. \%), & 0 wt. \%, & Alumina: $40-70 \mathrm{~nm}$ \\
DCAL-5 & TEGDMA (45 wt. \%), & 5 wt. \% & spherical, density \\
DCAL-10 & (2\% BHT), CQ (0.2 wt. \%) & 10 wt. \%, $3.95 \mathrm{~g} / \mathrm{ml}$ \\
DCAL-15 & and DMAEMA (0.8 wt. \%) & 15 wt. \%, \\
DCAL-20 & 20 wt. \% \\
DCMD-5 & 5 wt. \%, & Marble dust \\
DCMD-10 & 10 wt. \%, powder: $20-30$ \\
DCMD-15 & 15 wt. \%, $\mu$ m, density 2.6 \\
DCMD-20 & 20 wt. \% $\mathrm{g} / \mathrm{cm}^{3}$ \\
\hline
\end{tabular}




\subsection{Wear characterization}

Ducom dental wear simulator (Ducom, Bangalore, India) was used to evaluate the tribological characterization in the presence of an artificial saliva environment. Dental wear simulator is compatible to conduct tests according to the ASTM F732 test procedure. It was used to find the influence of various parameters such as normal load, sliding speed, and number of cycles on the tribological behavior of dental materials. The dental wear simulator is a single system that can characterize materials simultaneously at four multiple stations. All stations have the same identical complex movement pattern in two directions ( $\mathrm{X}$ and $\mathrm{Y}$, mutually orthogonal to the loading direction) over the antagonist disc made of SS-302 (Figure 1).

\subsection{Experimental design}

The Taguchi method is used to reduce the large number of iterations to a few steps. Instead of having to test all the possible combinations, the Taguchi method tests pairs of combinations and gives the optimum result. This reduces the number of experiments, time, and money. The volumetric wear of nanoalumina and micro-marble dust-filled dental composites were determined using a combination of four factors, each having five levels, and are presented in Table 5. The number of experiments involved was large in number as the effect of each factor on each variable was to be determined. Using the experimental approach, the impact of the four factors and five variables can be studied using the L25 orthogonal array. Using the L25 orthogonal array, the number of iterations evolved was reduced to 25 . The $\mathrm{S} / \mathrm{N}$ ratio for the four factors and five variables was determined by the selected smaller is the better characteristic, as our aim was to reduce the specific wear rate to the minimum. Smaller is the better characteristic was used as Eq. (2).

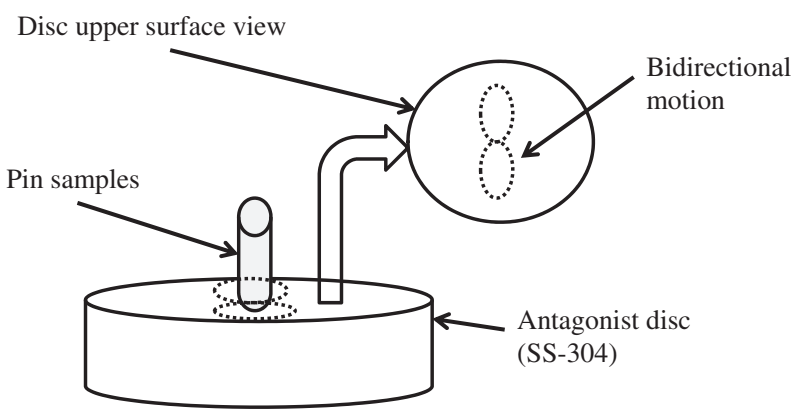

Figure 1: Block diagram of two-body abrasive dental wear.
Table 5: Levels of the variables used in the experiment.

\begin{tabular}{lrrrrrl}
\hline Control factors & I & II & III & IV & V & Units \\
\hline Sliding speed & 2.5 & 5 & 7.5 & 10 & 12.5 & $\mathrm{~mm} / \mathrm{s}$ \\
Normal load & 20 & 40 & 60 & 80 & 100 & N \\
Cycles & 4000 & 8000 & 12,000 & 16,000 & 20,000 & Cycles \\
Filler content & 0 & 5 & 10 & 15 & 20 & wt. \% \\
\hline
\end{tabular}

$$
\frac{\mathrm{s}}{\mathrm{N}}=-10 \log \frac{1}{\mathrm{n}}\left(\sum_{\mathrm{i}=2}^{\mathrm{n}} \mathrm{y}^{2}\right)
$$

where, $\mathrm{n}=$ the number of observations and $\mathrm{y}=$ the observed data, and $\mathrm{S} / \mathrm{N}=$ the signal-to-noise ratio.

\section{Results and discussion}

\subsection{Effect of hardness on nanoalumina and marble dust-filled dental composite}

The hardness of the dental composite filled with different wt. \% of nanoalumina and marble dust-filled dental composites are shown in the Figures 2 and 3, respectively. In Figure 2, it was observed that the unfilled dental composite DC-0 indicated a hardness value of $83 \mathrm{Hv}$, whereas the dental composite filled with 5 wt. \%, 10 wt. \%, 15 wt. \%, and 20 wt. \% nanoalumina exhibited hardness values of $94 \mathrm{Hv}, 116 \mathrm{Hv}, 140 \mathrm{Hv}$, and $154 \mathrm{Hv}$, respectively. On the other hand, Figure 3 indicated that the hardness value of the dental composite filled with 5 wt. \%, 10 wt. \%, 15 wt. \%,

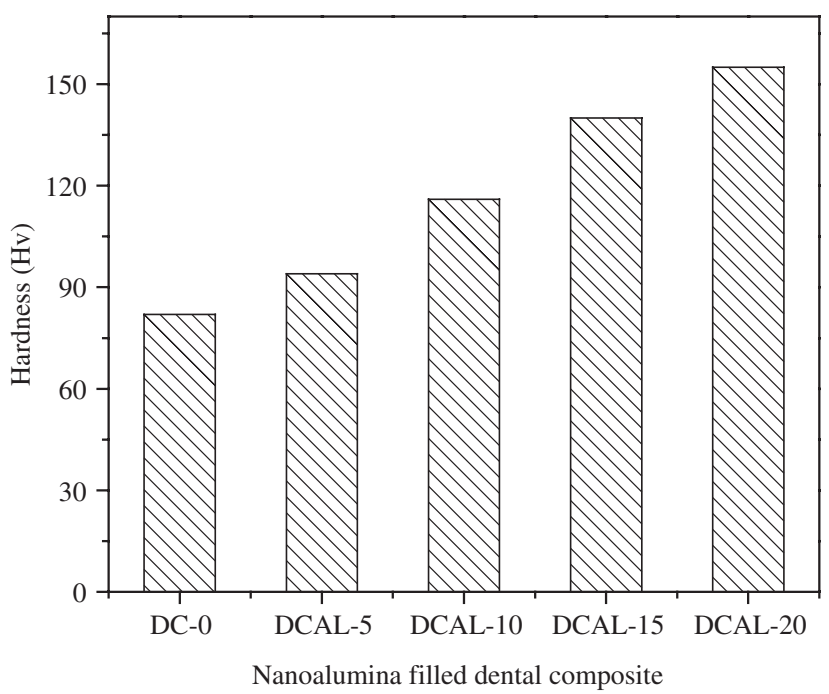

Figure 2: Effect of Vickers hardness on the nanoalumina-filled dental composite. 


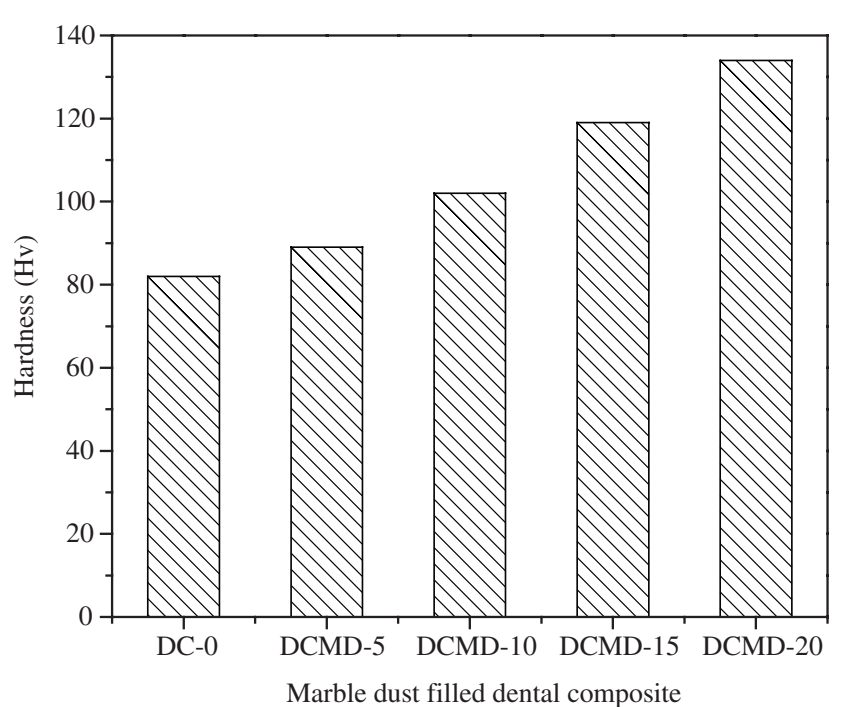

Figure 3: Effect of Vickers hardness on the marble dust-filled dental composite.

and 20 wt. \% marble dust was found to be $89 \mathrm{Hv}, 102 \mathrm{Hv}$, $119 \mathrm{Hv}$, and $134 \mathrm{Hv}$, respectively. In both the figures, it can be revealed that hardness increased with an increase in filler content. The increase in hardness with the increase in filler content was attributed to the fact that an increase in the hard and brittle ceramic filler such as nanoalumina and marble dust stiffened the elasticity of the matrix material and improved the material resistance to indentations on the surface of the materials. In both Figures 2 and 3, it was also observed that the addition of 5 wt. \% of nanoalumina increased the hardness of dental composite by $12 \%$, whereas an addition of 5 wt. \% of marble dust increased the hardness of the dental composite by 5 wt. \%. More hardness of the nanoalumina-filled composite compared to the marble dust-filled composite was attributed to a stronger binding of nanoalumina with silane compared to marble with silane. It can be also attributed to the fact that a decrease in filler size led to the improvement of the dispersion of the filler in the resin, which resulted in stronger interfacial bonding between the filler and the resin. This result was in agreement with the work by Borges et al. [31] in which it was proposed that nanocomposite and nanohybrid composite resins possess better Vickers microhardness than hybrid and micro-hybrid resins. They further concluded that the incorporation of nano-sized filler particles enhanced the mechanical properties of the dental composites. The increase in surface hardness with the increase in filler loading was in agreement with Kumar et al. [32]. However, the major factor affecting the hardness of the dental composite is the depth of cure of the resins, which depends upon various factors such as source of light polymerization, light intensity, exposure period, and irradiation distance [33, 34].

\subsection{Effect of a normal load on the volumetric wear of nanoalumina and marble dust- filled dental composites}

The effect of variation of the normal load on the volumetric wear of nanoalumina and marble dust dental composite is depicted in Figures 4 and 5, respectively. The steady state experiments were performed by varying the normal load from $20 \mathrm{~N}$ to $100 \mathrm{~N}$ in the step of $20 \mathrm{~N}$ under constant sliding speed of $5 \mathrm{~mm} / \mathrm{s}$ and number of cycles of 8000 . From both the figures, it can be revealed that an increase in load increased the volumetric wear of dental composites. Simultaneously, the increase in filler content led to a decrease in the volumetric wear of the dental composite.

Figure 4 indicated that for the unfilled composite, an increase in load from $20 \mathrm{~N}$ to $100 \mathrm{~N}$ increased the volumetric wear by $18.97 \%$, whereas in the case of a 20 wt. $\%$ nanoalumina-filled dental composite, an increase in load from $20 \mathrm{~N}$ to $100 \mathrm{~N}$ increased the volumetric wear by $52.38 \%$. Therefore, an increase in volumetric wear despite an increase in hardness in the 20 wt. \% + nanoaluminafilled composite may be attributed to the fact that due to the increase in load, the removed materials acted as a three-body abrasion along with a two-body abrasion.

Figure 5 indicates that in comparison to the $18.97 \%$ increase in volumetric wear for the unfilled composite, the 20-wt. \% marble dust-filled dental composite indicated an increase in volumetric wear by $76.26 \%$ as a result of a load increase from $20 \mathrm{~N}$ to $100 \mathrm{~N}$. Again, the increase in wear rate of the composite despite more hardness may be attributed to the weaker interfacial binding force between the matrix and the resin. Later, these removed materials acted as a part of a three-body abrasion. Turssi et al. [35]

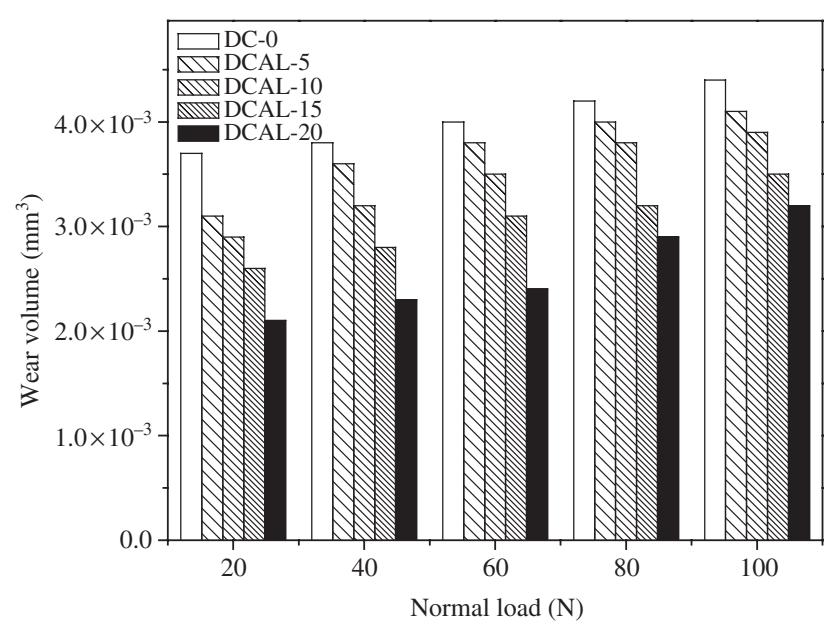

Figure 4: Effect of normal load on wear volume of the nanoaluminafilled dental composites. 


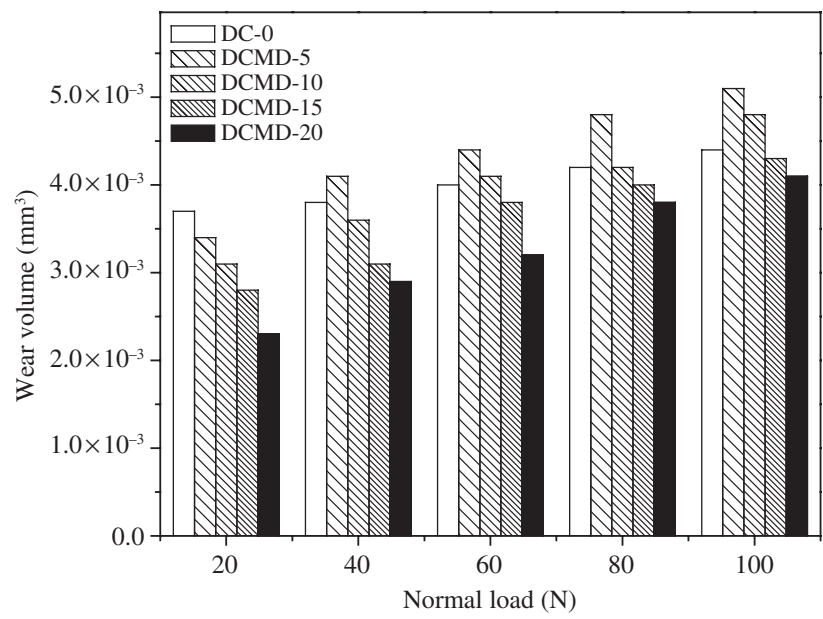

Figure 5: Effect of normal load on wear volume of the marble dust-filled dental composites.

found that when a normal load was applied, adhesively bonded contact junctions might have been ruptured and detached, generating larger friction coefficients and material loss after many repeated passes of the load.

In this study, less volumetric wear of a nanoaluminafilled dental composite compared to a marble dust-filled composite was in agreement with the work of Say et al. [36]. They suggested that wear resistance was improved with a smaller filler particle size. A more closely packed structure was formed with a smaller particle size, which helped to protect the resin matrix for further erosion [37].

\subsection{Effect of sliding speed on volumetric wear of nanoalumina and marble dust-filled dental composites}

The effect of variation of sliding speed on the volumetric wear of nanoalumina and marble dust dental composite is depicted in Figures 6 and 7, respectively. The steady state experiments were performed by varying the sliding speed from $2.5 \mathrm{~mm} / \mathrm{s}$ to $12.5 \mathrm{~mm} / \mathrm{s}$ in the step of $2.5 \mathrm{~mm} / \mathrm{s}$ under constant normal load of $40 \mathrm{~N}$ and number of cycles of 8000 . In both the figures, it can be revealed that an increase in sliding speed increased the volumetric wear of dental composites. It was attributed to the fact that an increase in sliding speed increased the tangential thrust force. Hence, the volumetric wear of the dental composite increased. Increase in sliding speed also increased the friction between two surfaces and their surface roughness. Apart from the normal load and speed, the presence of the medium also affected the wear performance of the dental composite. It has been documented that the $\mathrm{pH}$

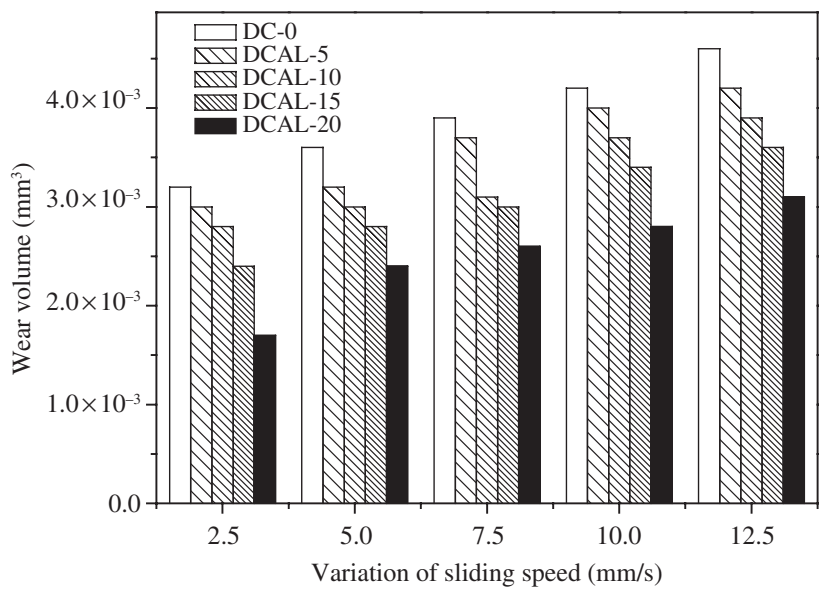

Figure 6: Effect of sliding speed on wear volume of the nanoalumina-filled dental composites.

of the working medium affected the degradation rates of the material through catalysis, being more unfavorable to hydrophilic resins [38]. Degradation of resin-based dental materials seems to progress at similar rates in water, artificial saliva, and in neutral to slightly low-pH media [39]. Figures 6 and 7 also indicated that for an unfilled composite, an increase in sliding speed from $2.5 \mathrm{~mm} / \mathrm{s}$ to $12.5 \mathrm{~mm} / \mathrm{s}$ increased the volumetric wear by $43.75 \%$, whereas in the case of a 20 -wt. \% nanoalumina-filled dental composite, it increased the volumetric wear by $23.52 \%$, and the 20-wt. \% marble dust-filled dental composite increased the volumetric wear by $29.03 \%$. Hence, it can be seen that apart from the applied load, an increase in sliding speed increased less volumetric wear of the nanoalumina-filled dental composite compared to the marble dust-filled dental composite.

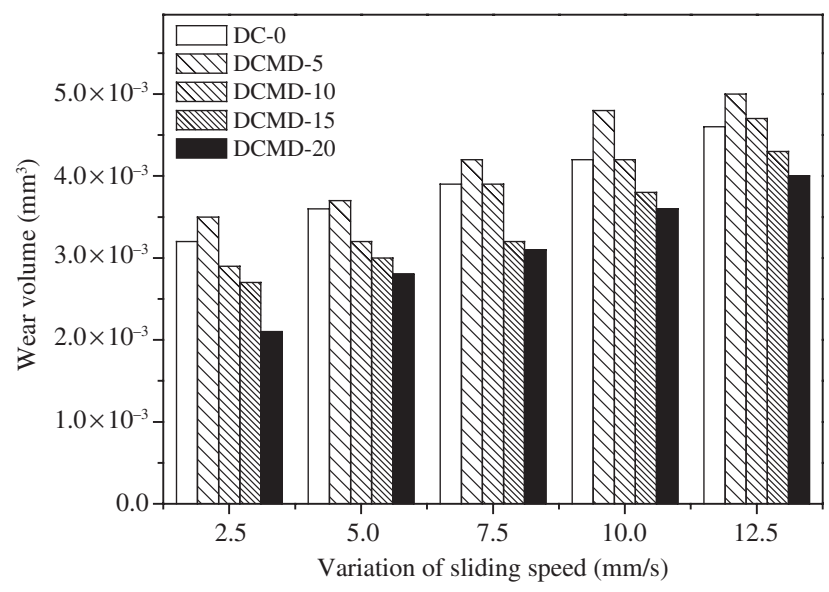

Figure 7: Effect of sliding speed on wear volume of the marble dust-filled dental composites. 


\subsection{Effect of number of cycles on the volumetric wear of nanoalumina and marble dust-filled dental composites}

The effect of variation of the number of cycles on the volumetric wear of nanoalumina and the marble dust dental composite are depicted in Figures 8 and 9, respectively. The steady state experiments were performed by varying the number of cycles from 4000 to 20,000 in the step of 4000 under a constant sliding speed of $5 \mathrm{~mm} / \mathrm{s}$ and a normal load of $40 \mathrm{~N}$. In both the figures, it can be revealed that an increase in the number of cycles increased the volumetric wear of the dental composites.

The increase in volumetric wear with the increase in the number of cycles was attributed to the fact that increasing the number of cycles increased the sliding distance and time period of the contact between the specimen and

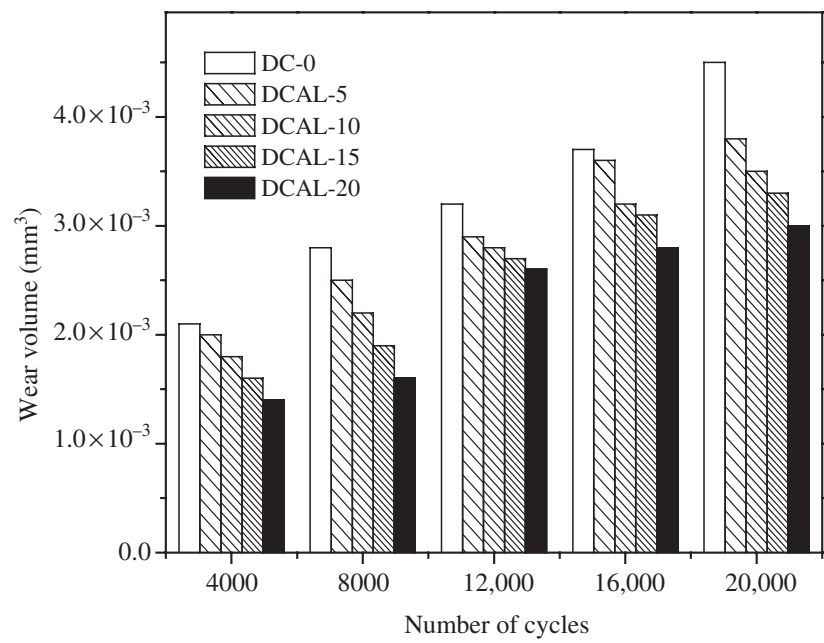

Figure 8: Effect of number of cycles on wear volume of the nanoalumina-filled dental composites.

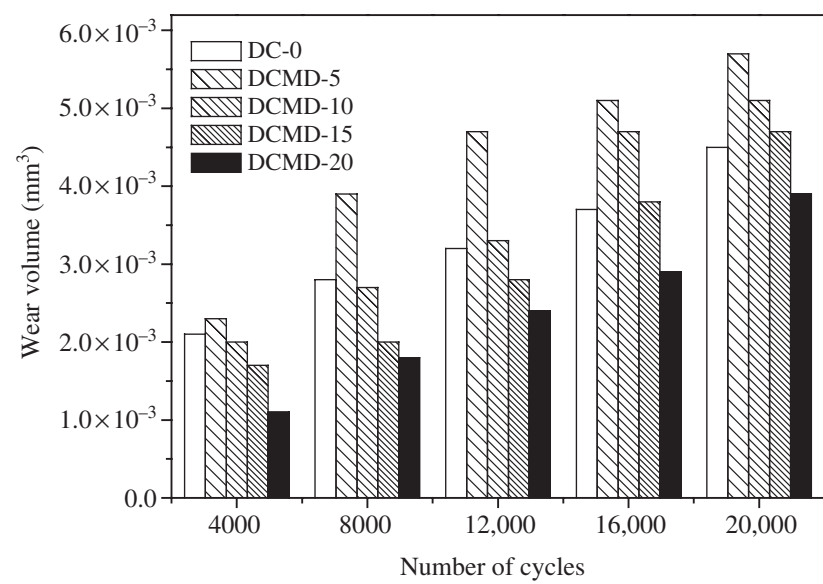

Figure 9: Effect of number of cycles on wear volume of the marble dust-filled dental composites. the antagonist disc. These results are in agreement with those reported by other investigators, which found that the volumetric wear in a dental composite increased linearly with the number of cycles, which was proportional to the sliding distance or sliding time [40]. Here, the wear resistant of the nanoalumina-filled composite was more than the marble dust-filled dental composite. It can be attributed to the fact that nano-sized particles occupy the interspacing between the resin matrix, which caused the reduction in erosion of the material. The more fine the particles are in the resin matrix, the more will be the wear resistance. The wear of large particles was more compared to the fine particles as they easily detach.

A study by Manhart et al. [41] showed that the wear of dental composites increased with an increase in the hardness, which is very different from the well-known relationship between wear and hardness. Other studies by Kon et al. [42] and Wonglamsam et al. [43] indicated that a simulated occlusal wear of a composite consisting of porous spherical filler was lower than that of the conventional composites despite the lower flexural strength of the former. These studies also indicated that the mechanical properties and wear of dental composites were not only the effect of filler content but also of filler morphology. Hence, in this regard, the wear of composites consisting of porous spherical filler would be differently influenced compared with that of conventional composites consisting of an irregular-shaped filler.

\subsection{Analysis of experimental results by the Taguchi experimental design}

The Taguchi experimental approach was applied to determine the dominant factors responsible for the overall reduction in volumetric wear of nanoalumina and marble dust-filled dental composites. The seventh and ninth columns in Table 6 indicate the $S / \mathrm{N}$ ratio of the nanoalumina- and marble dust-filled dental composites. The overall mean values for the $\mathrm{S} / \mathrm{N}$ ratio of the volumetric wear for the nanoalumina- and marble dustfilled dental composites were found to be $50.45 \mathrm{db}$ and $48.61 \mathrm{db}$, respectively. Also, the sixth and eighth columns in Table 6 indicated the volumetric wear of nanoalumina- and marble dust-filled dental composites as per the Taguchi orthogonal experimental design. It can be seen that the mean volumetric wear of the nanoaluminafilled dental composite $\left(0.00372 \mathrm{~mm}^{3}\right)$ exhibited $9.6 \%$ less wear compared to the marble dust-filled dental composite $\left(0.00408 \mathrm{~mm}^{3}\right)$. The results of the Taguchi experimental data for the nanoalumina- and marble dust-filled 
Table 6: Experimental design using the Taguchi $\mathrm{L}_{25}$ orthogonal array.

\begin{tabular}{|c|c|c|c|c|c|c|c|c|}
\hline S. no. & Filler (wt. \%) & Normal load (N) & Sliding speed (m/s) & No. of cycle & $\Delta \mathrm{V}_{\mathrm{AL}}\left(\mathrm{mm}^{3}\right)$ & $\mathrm{S} / \mathrm{N}$ ratio $(\mathrm{db})$ & $\Delta \mathbf{V}_{\mathrm{MD}}\left(\mathrm{mm}^{3}\right)$ & $\mathrm{S} / \mathrm{N}$ ratio $(\mathrm{db})$ \\
\hline 1 & 0 & 20 & 2.5 & 4000 & 0.001 & 60.00 & 0.002 & 53.98 \\
\hline 2 & 0 & 40 & 5.0 & 8000 & 0.003 & 50.46 & 0.004 & 47.96 \\
\hline 3 & 0 & 60 & 7.5 & 12,000 & 0.004 & 47.96 & 0.005 & 46.02 \\
\hline 4 & 0 & 80 & 10.0 & 16,000 & 0.005 & 46.02 & 0.005 & 46.02 \\
\hline 5 & 0 & 100 & 12.5 & 20,000 & 0.006 & 44.44 & 0.007 & 43.10 \\
\hline 6 & 5 & 20 & 5.0 & 12,000 & 0.005 & 46.02 & 0.005 & 46.02 \\
\hline 7 & 5 & 40 & 7.5 & 16,000 & 0.006 & 44.44 & 0.006 & 44.44 \\
\hline 8 & 5 & 60 & 10.0 & 20,000 & 0.003 & 50.46 & 0.006 & 44.44 \\
\hline 9 & 5 & 80 & 12.5 & 4000 & 0.002 & 53.98 & 0.002 & 53.98 \\
\hline 10 & 5 & 100 & 2.5 & 8000 & 0.001 & 60.00 & 0.001 & 60.00 \\
\hline 11 & 10 & 20 & 7.5 & 20,000 & 0.009 & 40.92 & 0.007 & 43.10 \\
\hline 12 & 10 & 40 & 10.0 & 4000 & 0.008 & 41.94 & 0.003 & 50.46 \\
\hline 13 & 10 & 60 & 12.5 & 8000 & 0.007 & 43.10 & 0.004 & 47.96 \\
\hline 14 & 10 & 80 & 2.5 & 12,000 & 0.006 & 44.44 & 0.003 & 50.46 \\
\hline 15 & 10 & 100 & 5.0 & 16,000 & 0.003 & 50.46 & 0.005 & 46.02 \\
\hline 16 & 15 & 20 & 10.0 & 8000 & 0.003 & 50.46 & 0.004 & 47.96 \\
\hline 17 & 15 & 40 & 12.5 & 12,000 & 0.002 & 53.98 & 0.005 & 46.02 \\
\hline 18 & 15 & 60 & 2.5 & 16,000 & 0.001 & 60.00 & 0.004 & 47.96 \\
\hline 19 & 15 & 80 & 5.0 & 20,000 & 0.002 & 53.98 & 0.005 & 46.02 \\
\hline 20 & 15 & 100 & 7.5 & 4000 & 0.001 & 60.00 & 0.006 & 44.44 \\
\hline 21 & 20 & 20 & 12.5 & 16,000 & 0.001 & 60.00 & 0.007 & 43.10 \\
\hline 22 & 20 & 40 & 2.5 & 20,000 & 0.002 & 53.98 & 0.001 & 60.00 \\
\hline 23 & 20 & 60 & 5.0 & 4000 & 0.003 & 50.46 & 0.001 & 47.96 \\
\hline 24 & 20 & 80 & 7.5 & 8000 & 0.004 & 47.96 & 0.001 & 60.00 \\
\hline \multirow[t]{2}{*}{25} & 20 & 100 & 10.0 & 12,000 & 0.005 & 46.02 & 0.004 & 47.96 \\
\hline & & & & & 0.00372 & & 0.00408 & \\
\hline
\end{tabular}

$\Delta \mathrm{V}_{\mathrm{AL}}$, Volumetric wear of nanoalumina-filled dental composite.

$\Delta \mathrm{V}_{\mathrm{MD}}$, Volumetric wear of marble dust-filled dental composite.

dental composites were analyzed using MINITAB 17 and are presented in Figures 10 and 11, respectively. Figure 10 indicated that the combination of factors should be in the form of A4, B5, C1, D1, and it would give a minimum volumetric wear for the dental composite filled with different wt. \% of nanoalumina. It means that the dental composite filled with $15 \mathrm{wt}$. \% nanoalumina at a normal load of $100 \mathrm{~N}$, with sliding speed of $2.5 \mathrm{~mm} / \mathrm{s}$ and number of cycles of 4000, exhibited a minimum wear rate. On the other hand, Figure 11 indicates that the combination of factors should be A4, B4, C1, D1, and it would give the dental composite filled with different wt. \% of marble dust a minimum volumetric wear. It means that the dental composite filled with $20 \mathrm{wt}$. \% of marble dust at a normal load of load of $80 \mathrm{~N}$, with sliding speed of $2.5 \mathrm{~mm} / \mathrm{s}$ and number of cycles of 4000, exhibited minimum wear rate.

\subsection{ANOVA and the effects of factors}

A general linear model analysis of variance (ANOVA) was performed to find the significance of factors such as filler content, normal load, sliding speed, and number of cycles on the volumetric wear of dental composites. ANOVA was performed based on the Taguchi experimental results. Tables 7 and 8 shows the results of the ANOVA with the volumetric wear of nanoalumina- and marble dust-filled dental composites taken from the Taguchi experimental design. This analysis was undertaken for a level of confidence of significance of $5 \%$. The last columns in Tables 7 and 8 indicate the percentage contribution of each factor. The percentage contribution indicates that the main effects are highly significant if the corresponding value of the percentage contribution is low. In Table 7, it can be observed that for the nanoalumina-filled dental composites, the level of significance follows the order filler content $(p=0.055)$, sliding speed $(p=0.138)$, number of cycles $(p=0.402)$, and normal load $(p=0.798)$. On the other hand, in Table 8 , it can be observed that for the marble dust-filled dental composites, the level of significance follows the order number of cycles $(p=0.037)$, filler content $(p=0.043)$, sliding speed $(p=0.066)$, and normal load ( $p=0.453)$. Hence, it can be revealed that in the case of a nanoalumina-filled dental composite, the filler content 


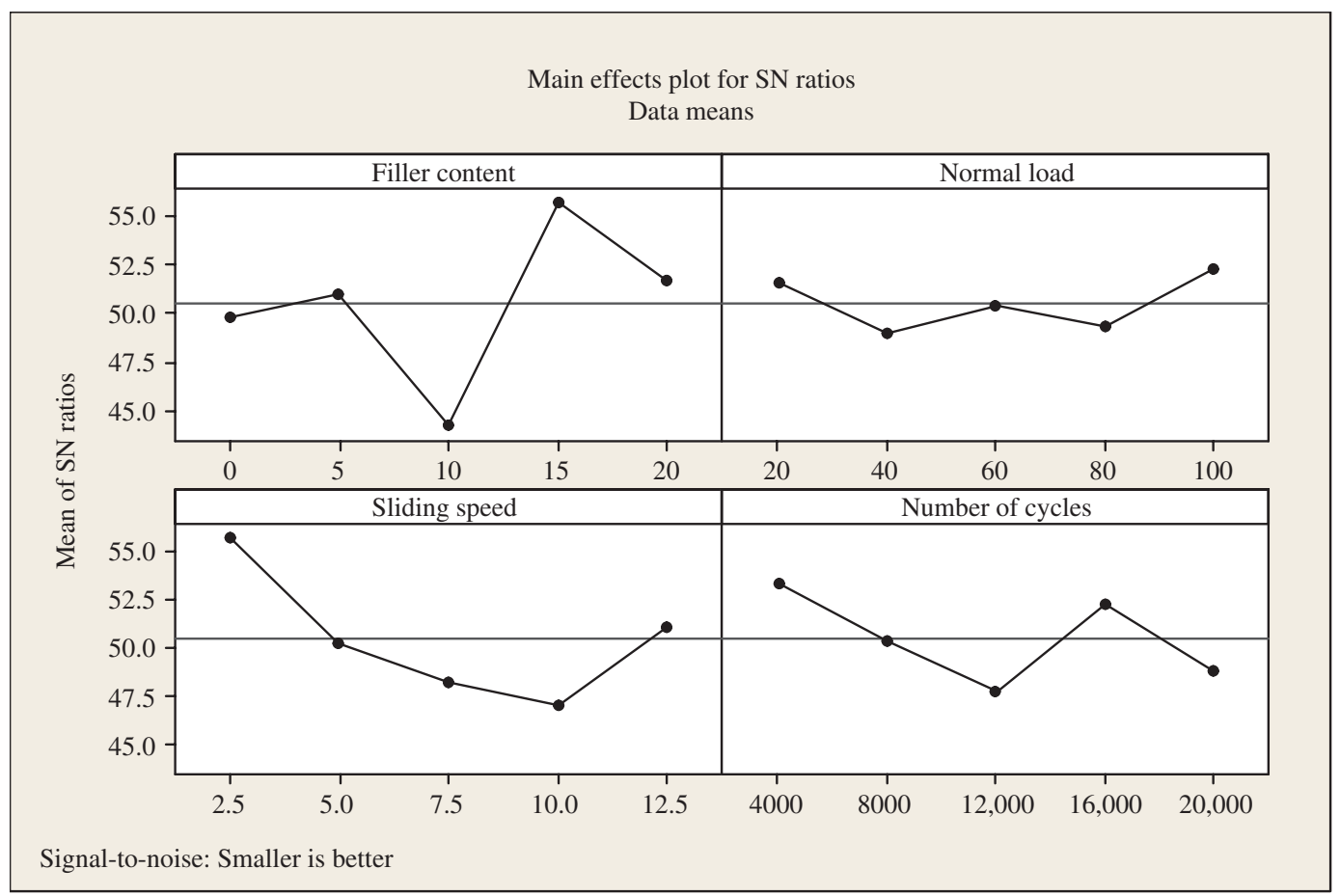

Figure 10: Effect of control factors on the wear rate of nanoalumina-filled dental composites.

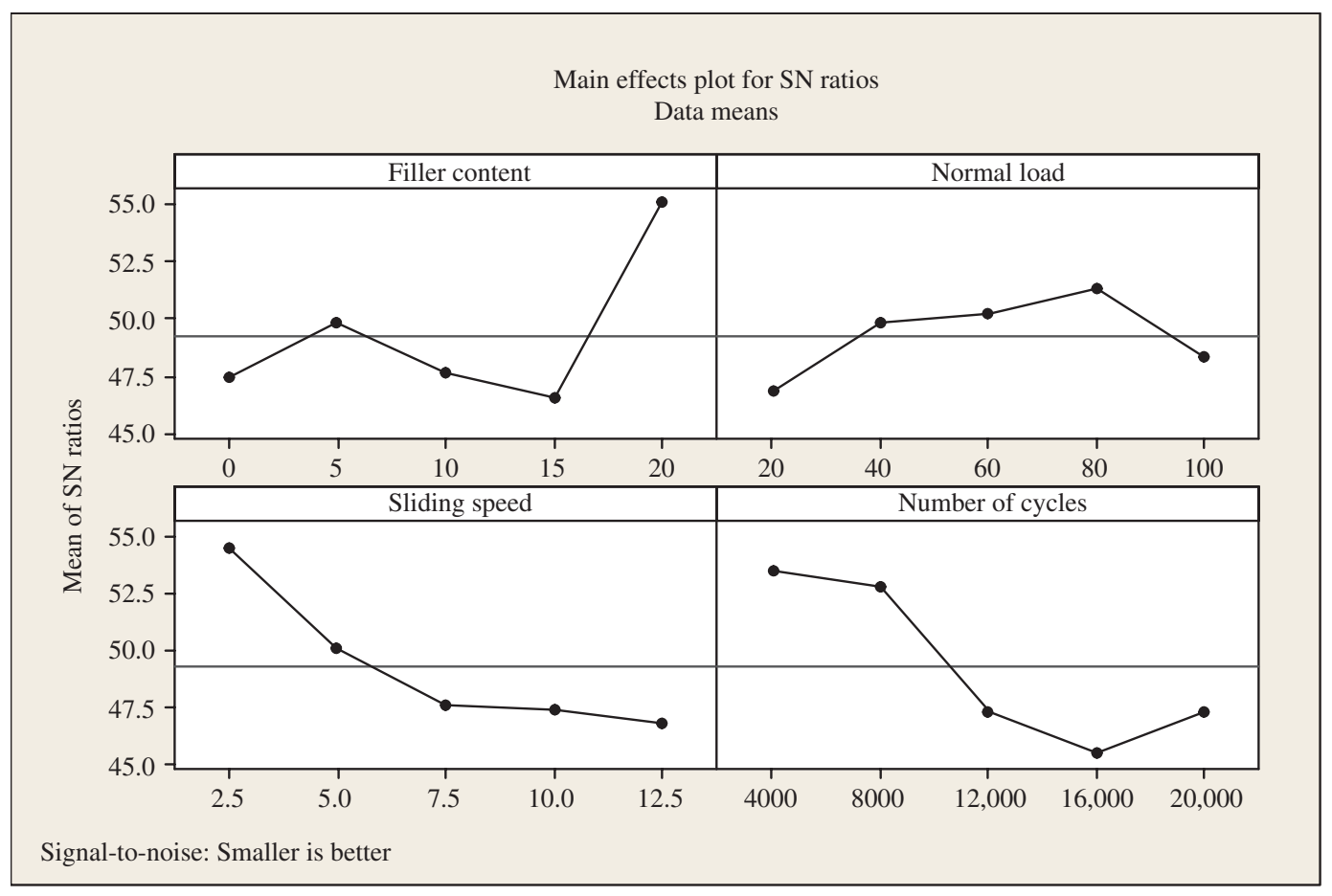

Figure 11: Effect of control factors on the wear rate of marble dust-filled dental composites.

is the most significant factor, whereas in the case of the marble dust-filled dental composite, the number of cycles is the most significant factor influencing volumetric wear of the dental composite. Hence, it can be concluded that as far as mechanical and wear properties are concerned, nanoalumina should be preferred over marble dust as a filler for the dental composite. Among all the factors, a normal load is the least significant factor. 
Table 7: ANOVA table for specific wear volume of nanoaluminafilled dental composite.

\begin{tabular}{lrrrrrr}
\hline Source & DF & Seq SS & Adj SS & Adj MS & F & P \\
\hline Filler content (wt. \%) & 4 & 345.46 & 345.46 & 86.37 & 3.67 & 0.055 \\
Normal load (N) & 4 & 38.35 & 38.35 & 9.59 & 0.41 & 0.798 \\
Sliding speed (m/s) & 4 & 223.55 & 223.55 & 55.89 & 2.38 & 0.138 \\
Cycles (rpm) & 4 & 107.60 & 107.60 & 26.90 & 1.14 & 0.402 \\
Error & 8 & 188.04 & 188.04 & 23.50 & & \\
Total & 24 & 902.99 & & & & \\
\hline
\end{tabular}

DF, Degree of freedom; Seq SS, sequential sum of square; Adj SS, adjacent sum of square; Adj MS, adjacent sum of mean square; $F$, variance; $P$, percentage contribution.

Table 8: ANOVA table for specific wear volume of marble dust-filled dental composite.

\begin{tabular}{lrrrrrr}
\hline Source & DF & Seq SS & Adj SS & Adj MS & F & P \\
\hline Filler content (wt. \%) & 4 & 241.26 & 241.26 & 60.31 & 4.08 & 0.043 \\
Normal load (N) & 4 & 60.19 & 60.19 & 15.05 & 1.02 & 0.453 \\
Sliding speed (m/s) & 4 & 200.88 & 200.88 & 50.22 & 3.39 & 0.066 \\
Cycles (rpm) & 4 & 258.23 & 258.23 & 64.56 & 4.36 & 0.037 \\
Error & 8 & 118.36 & 118.36 & 14.79 & & \\
Total & 24 & 878.91 & & & & \\
\hline
\end{tabular}

DF, Degree of freedom; Seq SS, sequential sum of square; Adj SS, adjacent sum of square; Adj MS, adjacent sum of mean square; $\mathrm{F}$, variance; $\mathrm{P}$, percentage contribution.

\subsection{Surface morphology}

The worn surface morphology of the nanoalumina- and marble dust-filled dental composites studied under steady state conditions using a field emission scanning electron microscope (FE-SEM) is presented in Figures 12 and 13, respectively. FE-SEM was used to characterize the morphology of unworn and worn surfaces of dental composites. Figure 12 indicates the unworn and worn surface morphology of the nanoalumina-filled dental composite under varying conditions of filler content, load, sliding speed, and number of cycles. Figure 12A shows the unworn surface of a 20-wt. \% nanoalumina-filled dental composite (DCAL20). It can be seen that there were a number of uneven and rough faces, voids, and cracks on the surface of the dental composite. Figure 12B and $\mathrm{C}$ indicates the effect of varying normal loads on the volumetric wear of the dental composite (DCAL-20). Figure 12B indicates the micro-graph of the surface at 20-N normal load, whereas Figure $12 \mathrm{C}$ indicates the micro-graph of a surface at 100-N normal load. It can be observed in both figures that at initial loading, wear track was not visible, but still, there was presence of wear scars and micro-ploughing, which was due to the presence of an uneven surface before the wear test. However, when the load was maximum at $100 \mathrm{~N}$, wear tracks were visible, and the surface was smooth. Therefore, an increase in load increased the volumetric wear, but wear rate was not significant, which is in agreement with our Taguchi result with the least normal load compared to other factors (Figure 10) and the steady state result (Figure 4).

Figure 12D and $\mathrm{E}$ indicates the effect of varying sliding speeds on the volumetric wear of a dental composite (DCAL-20). Figure 12D indicates a micro-graph of a surface at $2.5-\mathrm{mm} / \mathrm{s}$ sliding speed, whereas Figure 12E indicates a micro-graph of a surface at a sliding speed of $12.5 \mathrm{~mm} / \mathrm{s}$ keeping the other factors constant. At a low speed (Figure 12D), the filler particles were exposed and loosened on the surface, and delamination of the upper surface can be seen, but at maximum speed (Figure 12E), the removed particles participated in the three-body abrasion. More wear can be seen at high speed. Figure $12 \mathrm{~F}$ and $\mathrm{G}$ indicates the effect of varying numbers of cycles on the volumetric wear of a dental composite (DCAL-20). Figure $12 \mathrm{~F}$ indicates a micro-graph of a surface at 4000 number of cycles, whereas Figure $12 \mathrm{G}$ indicates a micro-graph of a surface at 20,000 numbers of cycles. At 4000 cycles of the run, the worn surfaces show pores and wear scars on the surface. However, after 20,000 cycles of the run, the surfaces become damaged, and a two-body abrasion can be seen on the surface. Figure $12 \mathrm{H}$ and I indicates the effect of varying filler contents on the volumetric wear of a dental composite. Figure $12 \mathrm{H}$ indicates a micro-graph of an unfilled dental composite (DC-0), whereas Figure 12I indicates a micro-graph of a 10-wt. \% nanoalumina-filled dental composites (DCAL-10). As per Figure 12H, the unfilled composites indicate an interlayer debonding of the matrix creating a fracture on the surface. As per Figure 12I, there was less wear compared to the unfilled dental composite, but still wear scars, small pits, holes were visible.

Figure 13 indicates the unworn and worn surface morphology of a marble dust-filled dental composite (DCMD-20) under different varying conditions of filler content, load, sliding speed, and number of cycles. Figure 13A shows the unworn surface of a 20 -wt. \% marble dust-filled dental composite (DCMD-20). The fabricated surfaces show a large number of voids, holes, and cracks on the surface. Figure $13 \mathrm{~B}$ and $\mathrm{C}$ indicates the effect of varying normal loads on the volumetric wear of a 20 -wt. \% marble dustfilled dental composite (DCMD-20). Figure 13B indicates a micro-graph of a surface at 20-N normal load, whereas Figure $13 \mathrm{C}$ indicates a micro-graph of a surface at $100-\mathrm{N}$ normal load. It can be observed in both the figures that at 20-N load, wear tracks were visible on the surface, whereas in the same working condition, wear track was 
A

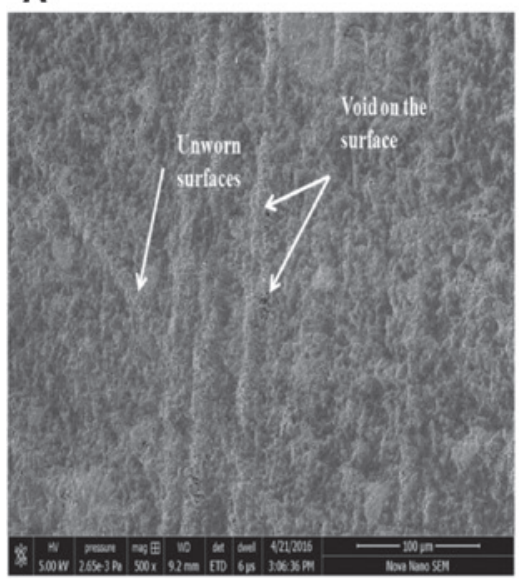

D

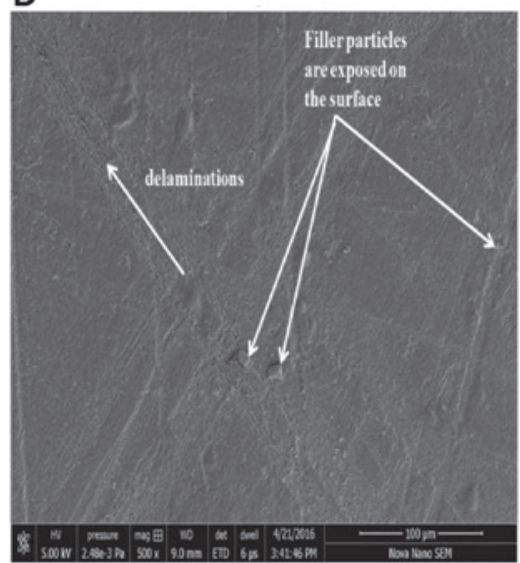

G

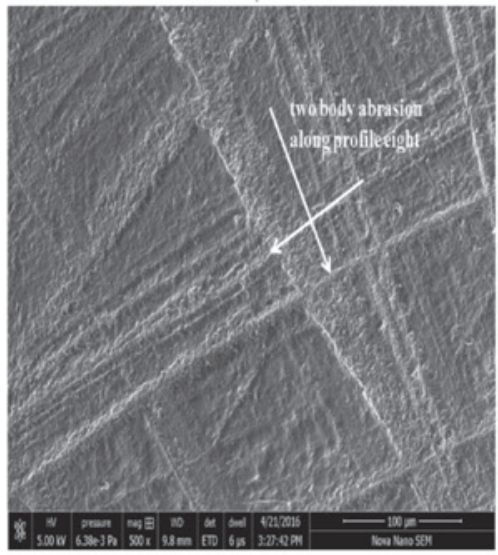

B

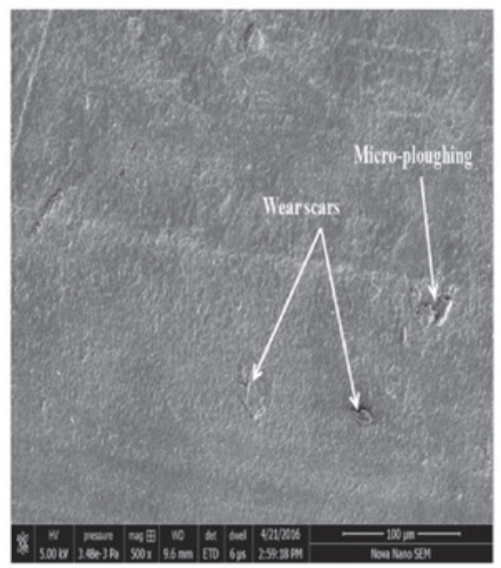

E

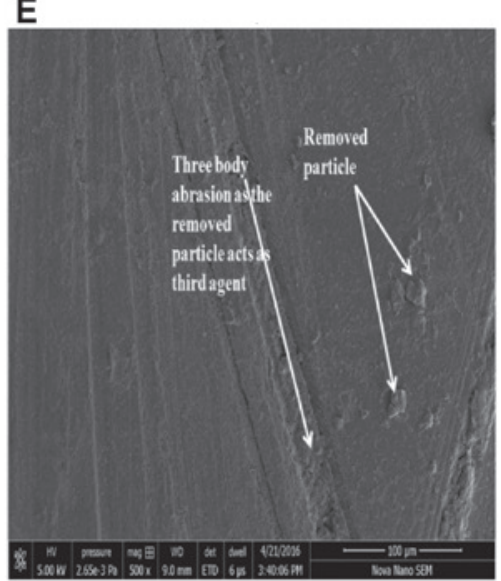

H

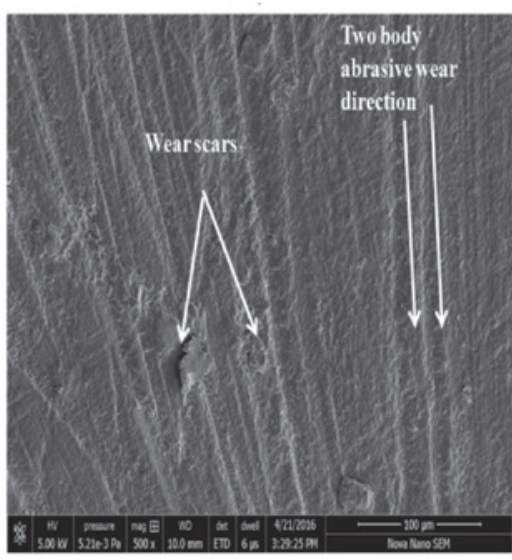

C

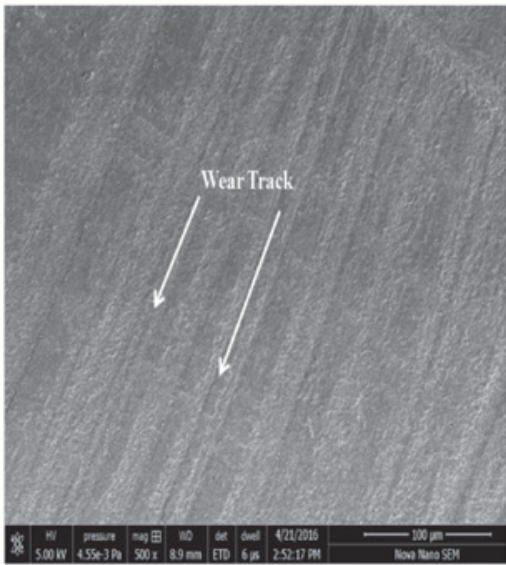

F

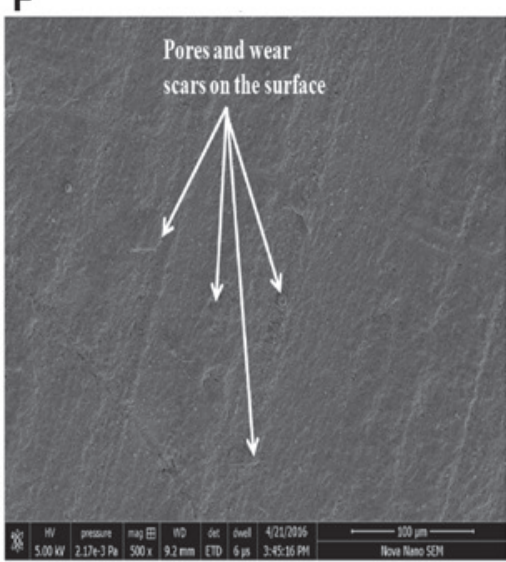

I

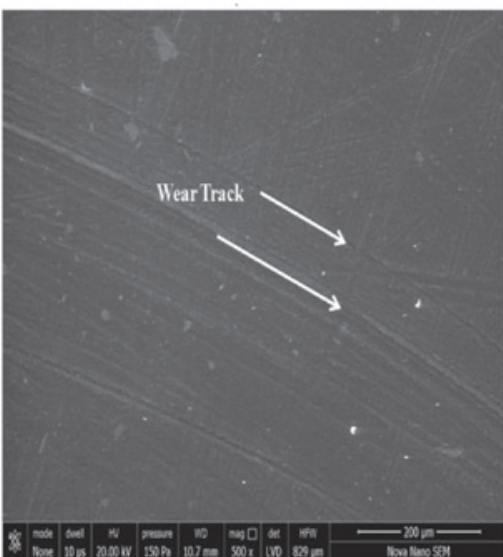

Figure 12: SEM of unworn and worn dental composites under steady state condition. (A) Dental composite DCAL-20 before wear test, (B) DCAL-20 at normal load $20 \mathrm{~N}$ (sliding speed at $5 \mathrm{~mm} / \mathrm{s}$ and number of cycles at 8000), (C) DCAL-20 at normal load $100 \mathrm{~N}$ (sliding speed at $5 \mathrm{~mm} / \mathrm{s}$ and number of cycles at 8000 ), (D) DCAL-20 at sliding speed $2.5 \mathrm{~mm} / \mathrm{s}$ (normal load $40 \mathrm{~N}$ and number of cycles at 8000 ), (E) DCAL-20 at number of cycles 4000 (sliding speed $2.5 \mathrm{~mm} / \mathrm{s}$ and normal load $40 \mathrm{~N}$ ), (F) DCAL-20 at number of cycles 20,000 (sliding speed $2.5 \mathrm{~mm} / \mathrm{s}$ and normal load $40 \mathrm{~N}$ ), (G) DCAL-0 at $40 \mathrm{~N}$ (sliding speed at $5 \mathrm{~mm} / \mathrm{s}$ and number of cycles at $8000,(\mathrm{H})$ DCAL-5 at $40 \mathrm{~N}$ (sliding speed at $5 \mathrm{~mm} / \mathrm{s}$ and number of cycles at 8000 ), (I) DCAL-10 at $40 \mathrm{~N}$ (sliding speed at $5 \mathrm{~mm} / \mathrm{s}$ and number of cycles at 8000 ).

not visible in nanoalumina-filled dental composites (DCAL-20) in Figure 12A. Hence, it can verify our result that the nanoalumina-filled dental composite exhibited better hardness compared to the marble dust-filled dental composite (DCMD-20). However, when the load was increased up to $100 \mathrm{~N}$ (Figure 13C), the worn surface was full of deep 
A

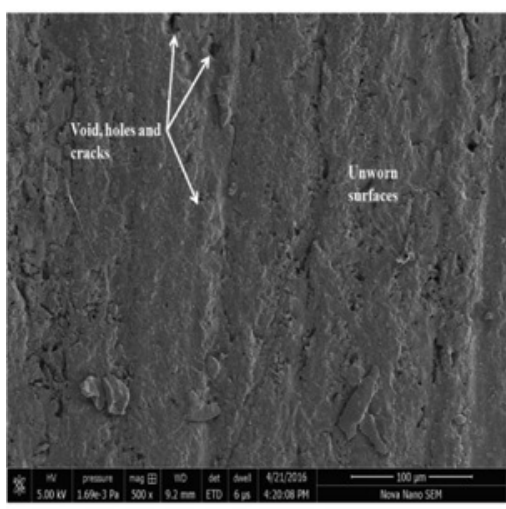

D

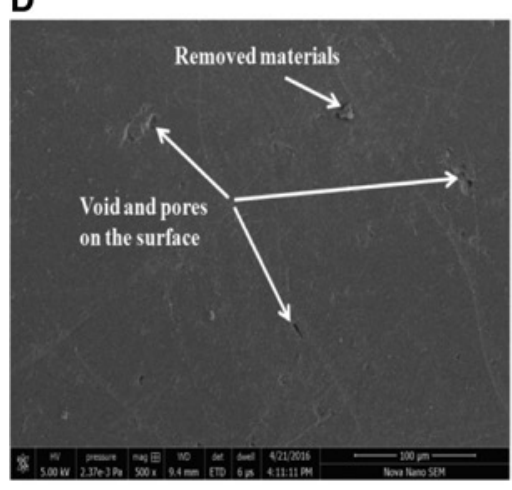

G

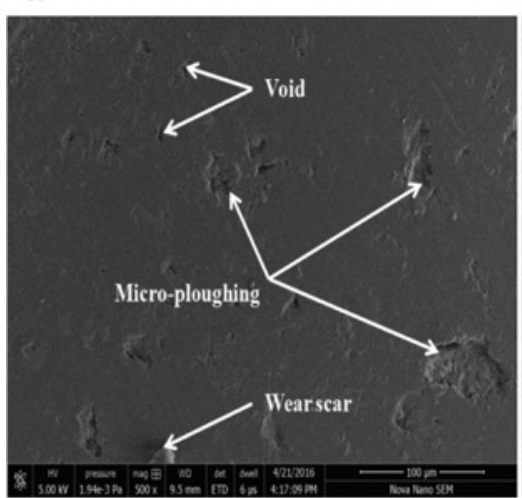

B

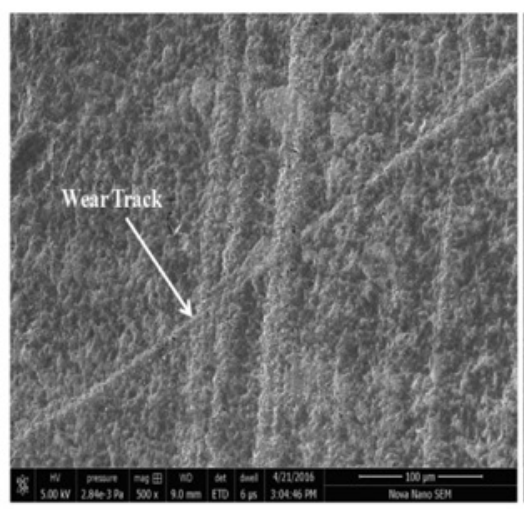

E

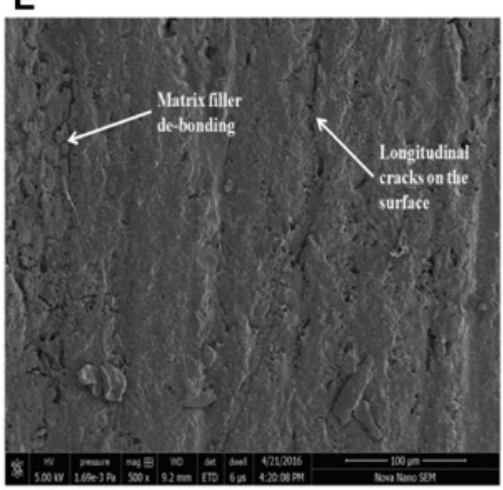

H

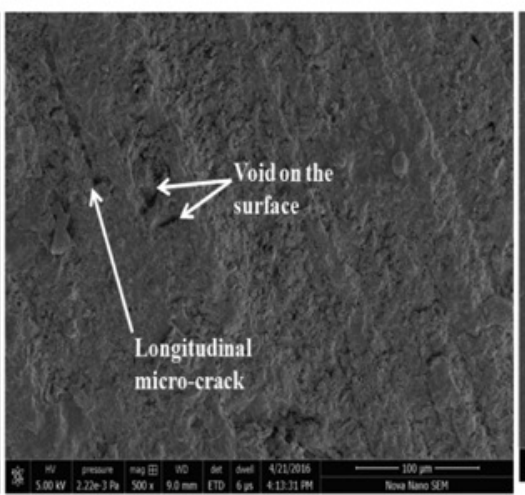

C

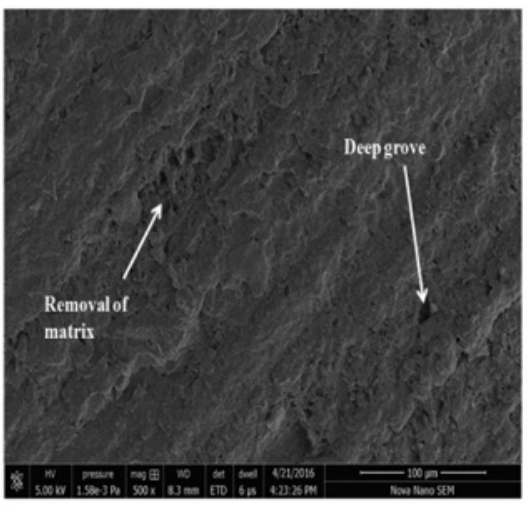

$\mathbf{F}$

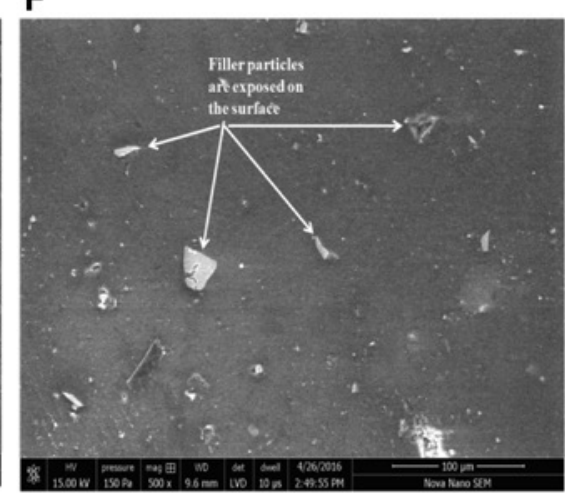

I

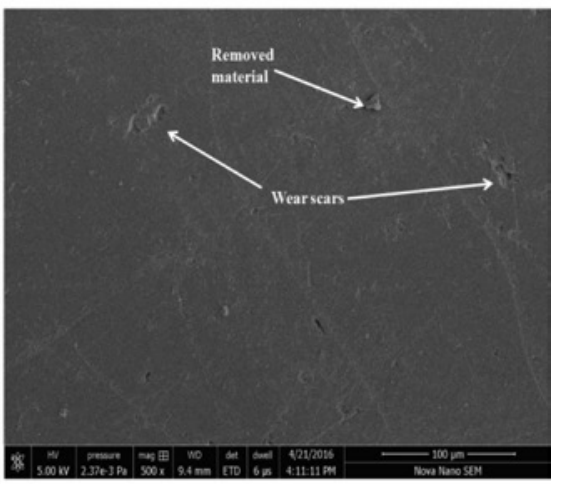

Figure 13: SEM of unworn and worn dental composites under steady state condition. (A) Dental composite DCMD-20 before wear test, (B) DCMD-20 at normal load $20 \mathrm{~N}$ (sliding speed at $5 \mathrm{~mm} / \mathrm{s}$ and number of cycles at 8000), (C) DCMD-20 at normal load $100 \mathrm{~N}$ (sliding speed at $5 \mathrm{~mm} / \mathrm{s}$ and number of cycles at 8000), (D) DCMD-20 at sliding speed $2.5 \mathrm{~mm} / \mathrm{s}$ (normal load $40 \mathrm{~N}$ and number of cycles at 8000 ), (E) DCMD-20 at sliding speed $12.5 \mathrm{~mm} / \mathrm{s}$ (normal load $40 \mathrm{~N}$ and number of cycles at 8000), (F) DCMD-20 at number of cycles 4000 (sliding speed $2.5 \mathrm{~mm} / \mathrm{s}$ and normal load $40 \mathrm{~N}$ ), (G) DCMD-20 at number of cycles 20,000 (sliding speed $2.5 \mathrm{~mm} / \mathrm{s}$ and normal load $40 \mathrm{~N}$ ), (H) DCMD-0 at (normal load $40 \mathrm{~N}$, sliding speed at $5 \mathrm{~mm} / \mathrm{s}$ and number of cycles at 8000, (I) DCMD-10 at $40 \mathrm{~N}$ ((normal load $40 \mathrm{~N}$, sliding speed at $5 \mathrm{~mm} / \mathrm{s}$ and number of cycles at 8000).

grooves, damaged surfaces, wear scars, etc. The delamination of the matrix material was also seen on the surface at a high loading of $100 \mathrm{~N}$.

Figure 13D and $\mathrm{E}$ indicates the effect of varying sliding speeds on the volumetric wear of a dental composite (DCMD-20). Figure 13D indicates a micro-graph of a surface at a $2.5-\mathrm{mm} / \mathrm{s}$ sliding speed, whereas Figure 13E indicates a micro-graph of a surface at a sliding speed of $12.5-\mathrm{mm} / \mathrm{s}$. At a low speed at $2.5 \mathrm{~mm} / \mathrm{s}$ (Figure 13D), the worn surface was smooth, interrupted by numerous cracks extending through the matrix. However, still, the removed materials were exposed on the surfaces along with some voids, which 
were left after exfoliation of the filler particles. As the speed was increased up to $12.5 \mathrm{~mm} / \mathrm{s}$ (Figure 13E), matrix filled debonding can be observed along with some longitudinal cracks perpendicular to the sliding direction. More wear can be seen at high speeds.

Figure $13 \mathrm{~F}$ and $\mathrm{G}$ indicates the effect of varying numbers of cycles on the volumetric wear of dental composites (DCMD-20). Figure 13F indicates a micro-graph of a surface at 4000 number of cycles, whereas Figure 13G indicates a micro-graph of a surface at 20,000 numbers of cycles. At 4000 cycles of run, the worn surfaces were smooth, but existence of voids, pores, and filler material can be seen on the surface. However, after 20,000 cycles of the run, the surfaces become damaged due to micro-ploughing by the two-body abrasion phenomena (Figure 13G).

Figure $13 \mathrm{H}$ and I indicates the effect of varying filler contents on the volumetric wear of dental composites. Figure $13 \mathrm{H}$ indicates a micro-graph of an unfilled dental composite (DC-0), whereas Figure 13I indicates a micrograph of 10 -wt. \% marble dust-filled dental composites (DCMD-10). As per Figure 13H, the unfilled composites indicated more wear compared to the 10 -wt. \% marble dust-filled dental composites in Figure $13 \mathrm{H}$ and the 20-wt. \% marble dust-filled composites in Figure 13B. The unfilled dental composite (Figure 13H) shows the presence of longitudinal crack and interlayer debonding of the matrix that led to create a fracture on the surface. As per Figure 13I, wear tracks were visible along with the presence of some wear scars and removed material.

\section{Conclusion}

The dental composites filled with different weight percentages of nanoalumina and marble dust were successfully fabricated. The Vickers hardness test of the dental composites was performed along with the two-body wear test in artificial saliva medium to assess their mechanical and wear properties. The hardness results indicated that the incorporation of $5 \mathrm{wt}$. $\%$ of nanoalumina increased the hardness of the dental composite by $12 \%$, whereas incorporation of $5 \mathrm{wt} . \%$ of marble dust increased the hardness of the dental composite by $7 \%$. The wear result indicated that the experimental composites based on nanoalumina and marble dust have been shown to behave differently in the artificial saliva medium. The nanoalumina-filled dental composites exhibited less volumetric wear compared to the marble dust-filled dental composite. In addition, other factors were found to play an important role in determining the wear performance of dental composites such as the filler content, applied normal load, sliding speed, and number of cycles.

The steady state condition tests indicated that the volumetric wear of dental composites increased with the increase in normal load and further increased with increasing number of cycles and sliding speed. The Taguchi orthogonal experiment suggested that for the nanoalumina-filled dental composite, the best combination of factors for minimum volumetric wear was the percentage of filler content of $15 \%$, normal load $100 \mathrm{~N}$, sliding speed $2.5 \mathrm{~mm} / \mathrm{s}$, and number of cycles 4000 . On the other hand, for the marble dust-filled dental composite, the best combination of factors for minimum volumetric wear was percentage of filler content at $20 \%$, normal load $80 \mathrm{~N}$, sliding speed $2.5 \mathrm{~mm} / \mathrm{s}$, and number of cycles 4000 . Further, it was also concluded that in the Taguchi orthogonal experiments, the mean volumetric wear of the nanoalumina-filled dental composite was 9.6\% less than that of the marble dust-filled dental composite in artificial saliva medium.

\section{References}

[1] Yap AU, Wee KE, Teoh SH, Chew CL. Oper. Dent. 2001, 26, 349-356.

[2] Hu X, Marquis PM, Shortall AC. J. Prosthet. Dent. 1999, 82, 214-220.

[3] Yap AU, Teoh SH, Chew CL. Dent. Mater. 2002, 18, 149-158.

[4] Shabanian M, Richards LC. J. Prosthet. Dent. 2002, 87, 650-656.

[5] de Gee AJ, Leloup G, Werner A, Vreven J, Davidson CL. J. Dent. Res. 1998, 77, 1658-1663.

[6] de Gee AJ, van Duinen RNB, Werner A, Davidson CL. J. Dent. Res. 1996, 75, 1613-1619.

[7] Yap AU, Teoh SH, Tan KB. J. Biomed. Mater. Res. 2000, 53, 547-553.

[8] de Gee AJ, Pallav P, Davidson CL. J. Dent. Res. 1986, 65, 654-658.

[9] Dickenson GL, Leinfelder KF. J. Am. Dent. Assoc. 1993, 124, 68-72.

[10] Settembrini L, Penugonda B, Fischer E. J. Clin. Dent. 1993, 4, 55-60.

[11] Hotta M, Hirukawa H. Oper. Dent. 1994, 19, 42-46.

[12] Tanoue N, Matsumura H, Atsuta M. J. Prosthet. Dent. 2000, 84, 93-97.

[13] Abenojar J, Martinez MA, Velasco F, Sanchez VP, MartínMartínez JM. J. Adhes. 2009, 85, 216-238.

[14] Abenojar J, Martinez MA, Velasco F, del Real JC. J. Adhes. Sci. Technol. 2011, 25, 2445-2460.

[15] Abenojar J, Martinez MA, Pantoja M, del Real JC. J. Adhes. 2012, $88,418-434$

[16] Willmann G. J. Mater. Process. Technol. 1996, 56, 168-176.

[17] Galindo ML, Sendi P, Marinello CP. J. Prosthet. Dent. 2011, 106, $23-28$. 
[18] Fischer H, Waindich A, Telle R. Dent. Mater. 2008, 24 , 618-622.

[19] Stump ASG, Bergmann CP, Vicenzi J, Fetter R, Mundstock KS. Mater. Des. 2009, 30, 4348-4359.

[20] Xia Y, Zhang F, Xie H, Gu N. J. Dent. 2008, 36, 450-455.

[21] Hamburger JT, Opdam NJ, Bronkhorst EM, Kreulen CM, Roeters JJ, Huysmans MC. J. Adhes. Dent. 2011, 13, 585-593.

[22] Deliperi S, Bardwell DN. J. Am. Dent. Assoc. 2002, 133, 1387-1398.

[23] Misra AK, Mathur R, Rao YV, Singh AP, Goel P. J. Sci. Ind. Res. (India) 2010, 69, 67-72.

[24] Baboo R, Khan NH, Kumar A, Tabin RS, Duggal SK. I.J.C.S.E. 2011, 1, 827-834.

[25] Saboya F, Xavier GC, Alexandre J. Constr. Build. Mater. 2007, 21, 1950-1960.

[26] Gangwar S, Patnaik A, Bhat IK. Silicon 2017, 9, 249-263.

[27] Kumar SR, Patnaik A, Bhat IK. J. Compos. Mater. 2017, 51, 1997-2008.

[28] Kelestemur O, Yildizs S. Sci. Iran. Trans. A: Civ. Eng. 2016, 23, 896-906.

[29] Kelestemur O, Anci E, Servet Y, Gokcer B. Constr. Build. Mater. 2014, 60, 17-24.

[30] Kelestemur O, Yildizs S, Gokcer B, Anci E. Mater. Des. 2014, 60, 548-555.
[31] Borges BC, Groninger Al, Soares GP, Santos-Daroz CBD, Ambrosano GMB, Marchi GM, Giorgi MCC, Aguiar FHB. Dentistry 2011, 103, 1-5.

[32] Kumar SR, Patnaik A, Bhat IK. P. I. Mech. Eng. L-J. Mat. 2016, 230, 492-503.

[33] Pires JA, Cvitko E, Denehy GE, Swift EJ. Quintessence Int. 1993 24, 517-521.

[34] Groninger AIS, Soares GP, Sasaki RT, Ambrosano GMB, Lovadino JR, Aguiar FHB. Brazilian J. Oral. Sci. 2011, 10, 189-192.

[35] Turssi CP, Ferracane JL, Vogel K. Biomaterials 2005, 26, 4932-4937.

[36] Say EC, Civelek A, Nobecourt A, Ersoy M, Guleryuz C. Oper. Dent. 2003, 28, 628-634.

[37] Clelland NL, Pagnotto MP, Kerby RE, Seghi RR. J. Prothet. Dent. 2005, 93, 153-157.

[38] Gopferich, A. Biomaterials 1996, 17, 103-114.

[39] Cilli R, Pereira JC, Prakki A. J. Dent. 2012, 40, 1144-1150.

[40] Callaghan DJ, Vaziri A, Hashemi HN. Dent. Mater. 2006, 22, 84-93.

[41] Manhart J, Kunzelmann KH, Hickel R. J. Biomed. Mater. Res. 2000, 53, 353-361.

[42] Kon M, Kakuta K, Ogura H. Dent. Mater. J. 2006, 25, 183-194.

[43] Wonglamsam A, Kakuta K, Ogura H. Dent. Mater. J. 2008, 27, 243-250. 